\title{
ROTURA TRAUMÁTICA DE URETRA FEMENINA: UNA RARA ENTIDAD. APORTACIÓN DE DOS CASOS
}

\author{
A. BLANCO DÍEZ, L. ÁlVAREZ CASTELO, E. FERNÁNDEZ ROSADO, \\ J. SÁNCHEZ RODRÍGUEZ-LOSADA, V. CHANTADA ABAL, S. NOVÁS CASTRO, \\ M. RUIBAL MOLDES, A. BARBAGELATA LÓPEZ, M. GONZÁLEZ MARTÍN
}

Servicio de Urología. Complejo Hospitalario Universitario Juan Canalejo. La Coruña.

Actas Urol Esp. 28 (4): 318-323, 2004

\section{RESUMEN}

ROTURA TRAUMÁTICA DE URETRA FEMENINA: UNA RARA ENTIDAD. APORTACIÓN

DE DOS CASOS

Presentamos dos casos clínicos de rotura de uretra femenina, una situación poco frecuente por las características anatómicas de la mujer pero que debe ser sospechada precozmente, ante todo politraumatismo pélvico, para evitar graves problemas.

Los casos expuestos son dos pacientes jóvenes politraumatizadas por accidentes de tráfico con fracturas óseas pélvicas. En ambas se realizó cirugía combinada suprapúbica-vaginal, una de ellas previo realineamiento endoscópico precoz. Esta precisó tratamiento endoscópico complementario: electrofulguración de pequeño orificio fistuloso. La evolución de ambas es buena con continencia.

Hacemos una revisión de la literatura.

PALABRAS CLAVE: Rotura uretral. Mujeres. Trauma pélvico.

\begin{abstract}
TRAUMATIC URETHRAL DISRUPTION IN WOMEN. A RARE ENTITY. APORTATION OF TWO CASES

We present two cases of urethral disruption in women, a very uncommon situation because of the anatomical features. Yet it must be always suspected in all women under a pelvic politraumatism, for eviting important problems.

Both cases are traffic politraumatized young girls presenting with pelvic fracture, among others. The surgery performed was combined suprapubic-transvaginal approach, even in one a previous endoscopic realinement was done, who also needed endoscopic complementary treatment: electrofulguration of a fistulous bridge.

Outcome of both is fine, achieving good continence.

We also make a literature review.
\end{abstract}

KEY WORDS: Urethral disruption. Women. Pelvic trauma.

$\mathrm{L}$ a rotura traumática de la uretra femenina es un acontecimiento poco habitual y poco documentado, debido básicamente a las características anatómicas de la uretra femenina. Se asocia sobre todo a grandes traumatismos pélvicos por accidentes de tráfico. Debe ser sospechada en toda mujer con el antecedente de un gran trauma pélvico que asocie: sangre en uretra-vagina, dificultad de sondaje, salida de orina por vagina, etc. No es raro encontrar otras lesiones perineales (por ejemplo, laceraciones anales). La sospecha y un correcto diagnóstico y manejo, pueden evitar complicaciones como las estenosis uretrales o vaginales, el hematocolpos en pacientes post-púberes, 
y problemas más graves como la enfermedad inflamatoria pélvica que pueda hacer necesaria una histerectomía. El manejo inicial es controvertido, habiendo grupos que preconizan la colocación de cistostomía suprapúbica y el tratamiento diferido, otros el sondaje y realineamiento endoscópico inicial, y otros el tratamiento primario. En cualquier caso existen cuatro factores que van a determinar el procedimiento a seguir: el estado clínico de la paciente, la severidad de la fractura púbica y la desestructuración anatómica, y la experiencia del equipo quirúrgico.

\section{CASOS CLÍNICOS}

Caso 1. Mujer de 25 años. Ingresa en el servicio de urgencias de otro centro en octubre de 1999 por politraumatismo por accidente de tráfico. Presenta fractura de articulaciones sacro-ilíacas y de ambas ramas públicas, lesiones incisas en cara anterior vaginal, y rotura traumática de uretra con apertura hacia cavidad vaginal. Inicialmente fue sondada saliendo la sonda por cara anterior de vagina. Se colocó talla vesical y la incisión vaginal fue suturada por ginecología.

Se remite a nuestro centro una vez estabilizada (3 meses más tarde) para valoración de tratamiento. A la exploración física se observa: estenosis completa de uretra a $1 \mathrm{~cm}$ de meato uretral. Pared anterior de vagina retraída y fibrótica a nivel de porción media de uretra. La cistografía realizada a través de la talla vesical muestra una vejiga normal, sin apertura de cuello y sin micción (Fig. 1).

Intervención quirúrgica: se realiza uretroplastia combinada transvaginal y suprapúbica percutánea. En posición de litotomía se observa cara anterior de vagina fibrosada con intensa cicatriz y retracción que engloba mitad proximal de uretra. El cuello vesical observado por cistoscopia suprapúbica anterógrada aparece cerrado. Se procede a apertura de cara anterior de vagina y liberación de uretra. Se abre la luz de la misma entrando en vejiga. Se coloca sonda vesical y se sutura la uretra alrededor con sutura reabsorbible. Se deja talla suprapúbica.

$1^{\text {a }}$ revisión: 3 meses tras intervención quirúrgica: no presenta incontinencia pero la flujometría es obstructiva, con flujo máximo de 3,4 $\mathrm{ml} / \mathrm{sg}$ y el residuo escaso $(20 \mathrm{cc}$ ). Se indica protocolo de dilataciones uretrales (Fig. 2).

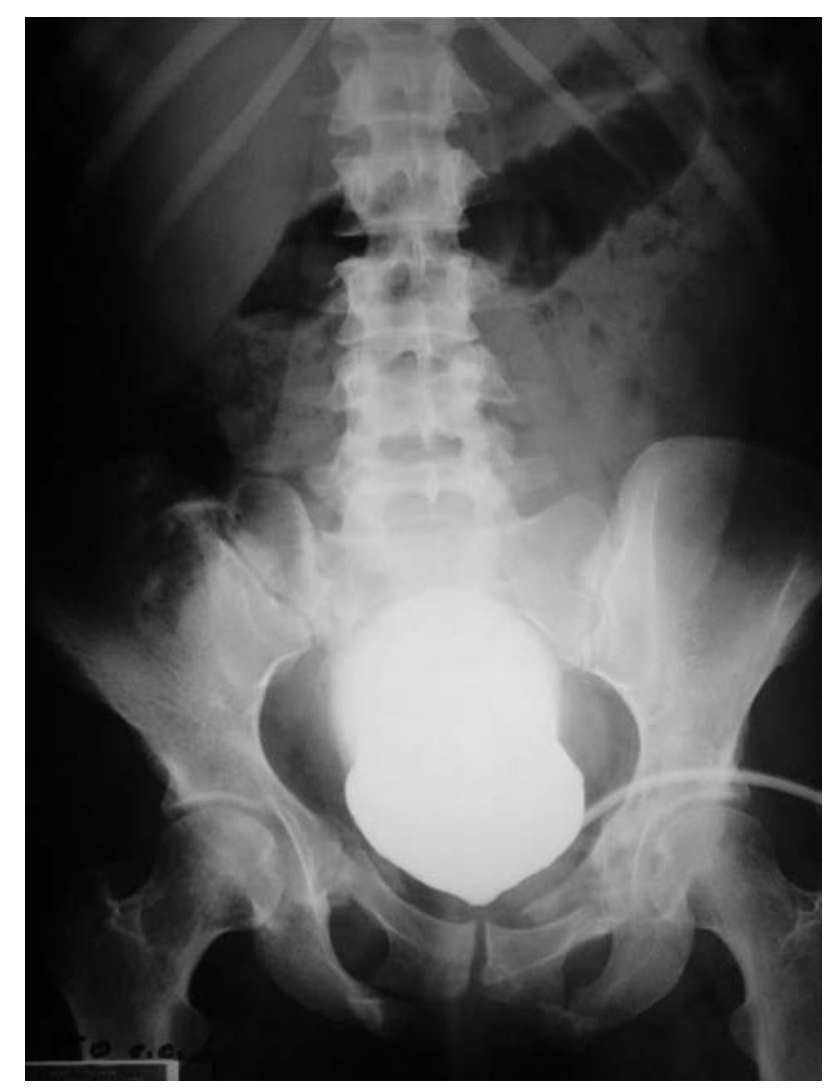

FIGURA 1. Cistografia por talla vesical. Se observa cuello vesical cerrado y ausencia de micción.

Ültima consulta y tras 2 años de seguimiento la paciente no presenta dificultad miccional, no-incontinencia, no-polaquiuria. Actividad sexual normal (embarazada). Sin necesidad de dilataciones.

Caso 2. Mujer de 17 años. Ingresa en U.C.I. de nuestro centro en noviembre de 2000 por politraumatismo por accidente de tráfico. Presenta trauma torácico, con fracturas costales y de clavícula. Fractura de pelvis y sacro.

Nos avisan 3 días más tarde para valorar sonda vesical puesta al ingreso y que fue recambiada varias veces (no por urólogo) por mal funcionamiento. Se realizan cistografia y TAC. Cistografia retrógrada: salida de contraste fuera de vejiga. Rotura vesical extraperitoneal. TAC.: extravasación de contraste en pelvis con rotura de vejiga y desinserción uretral (Fig. 3). Se coloca talla vesical y se realiza nueva cistografía por talla: rotura de uretra y cuello vesical. 22-11-00 cistoscopia: rotura de uretra anterior y rotura de cara anterior vesical. Sobre guía de 0,035 mm se coloca sonda tipo Foley. 


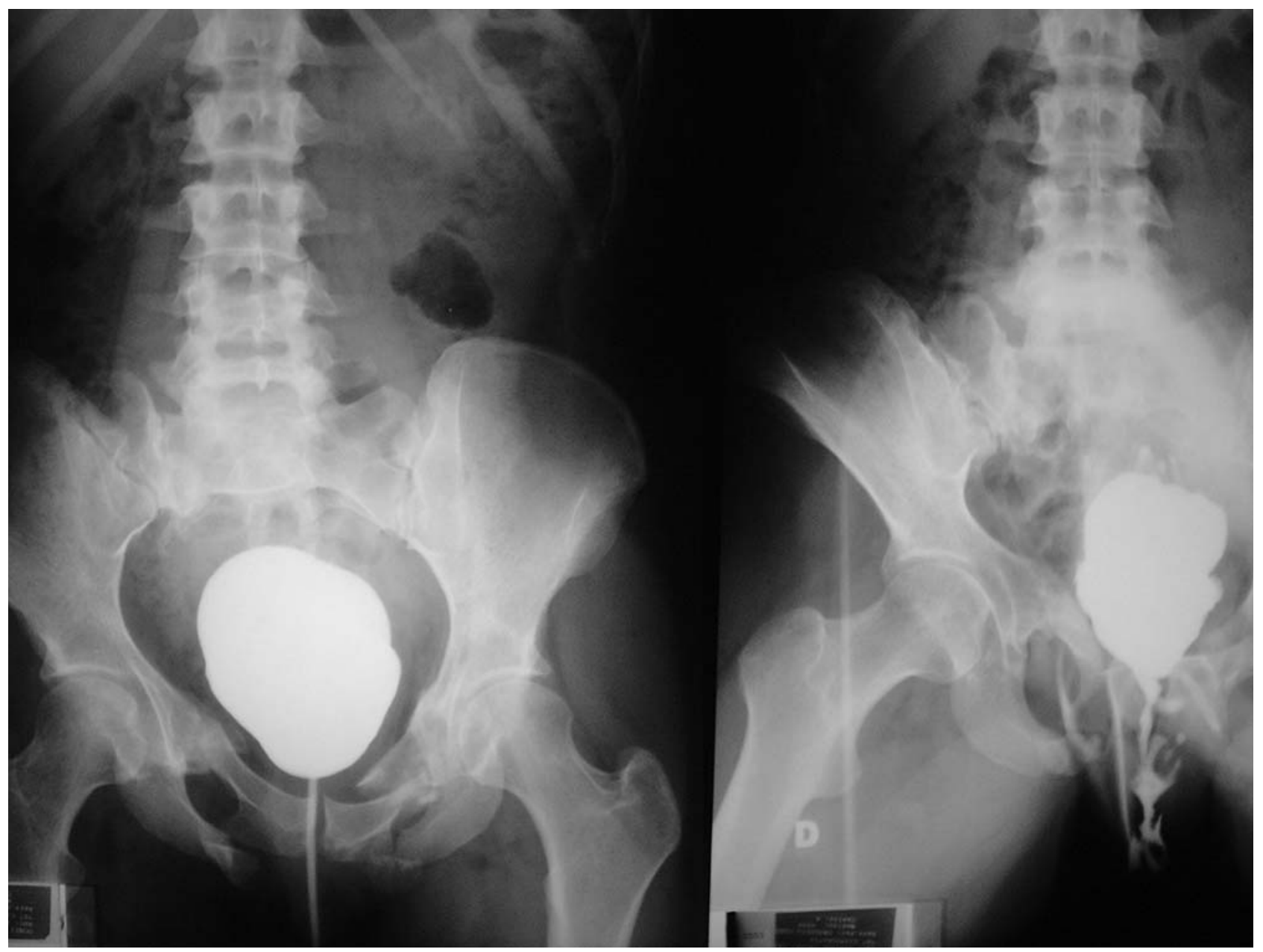

FIGURA 2. Cistografía retrógrada y miccional post-quirúrgicas. Se observa ausencia de fugas y micción espontánea.

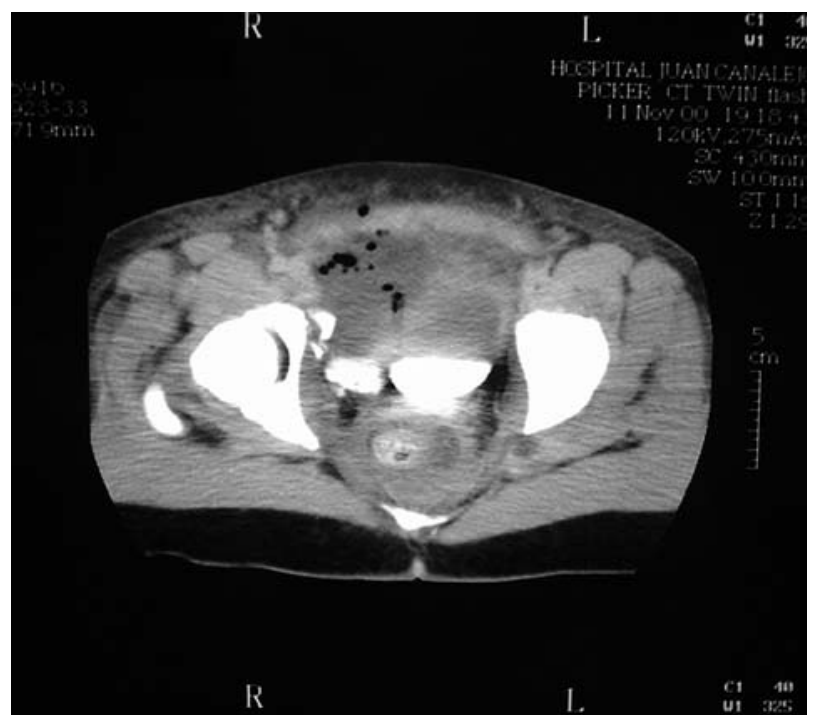

FIGURA 3. TAC: extravasación de contraste en pelvis. Rotura de vejiga y desinserción uretral.
Cistografia de control: fuga de contraste en cuello vesical.

Intervención quirúrgica. Uretroplastia con uretrocistoplastia. Se observa rotura de cara anterior vesical y rotura uretral con uretra desgarrada hasta $0,5 \mathrm{~cm}$ del meato uretral. Se observa un puente entre vejiga y uretra. Se realiza primero sutura de vejiga y después sutura de la uretra para lo cual es necesario un abordaje combinado suprapúbico-vaginal. La uretra se sutura a cara anterior de pubis en un intento de reconstruir anatomía (Figs. 4 y 5).

Cistouretrografía: fuga de contraste en uretra anterior.

Cirugia: electrofulguración de divertículo posttraumático uretral anterior. Resector y asa de Collins. Sonda vesical. 

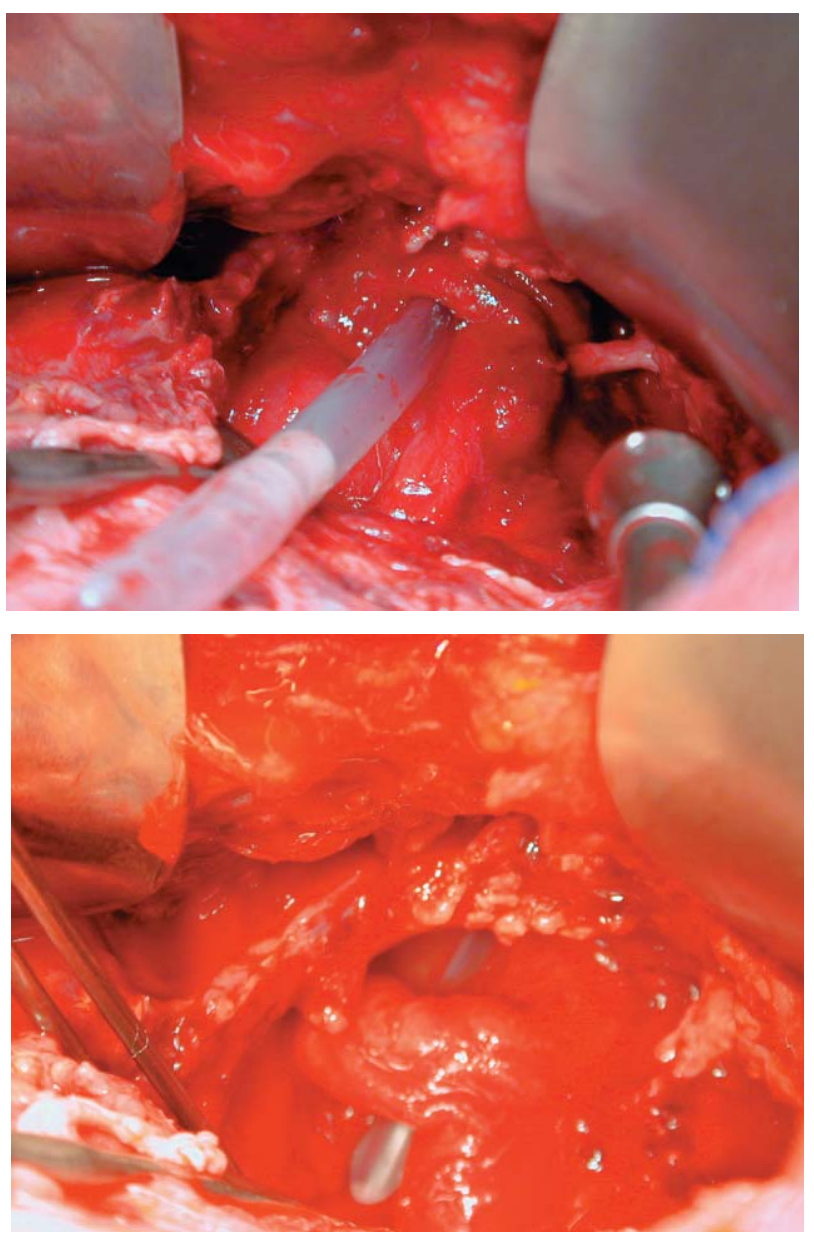

FIGURAS 4 y 5. Rotura vesical de cuello y uretra durante intervención.

Ultima consulta, 11-12-02: tras año y medio de seguimiento la paciente presenta una cistografía normal (Fig. 6). Un estudio urodinámico con flujo máximo de 15,6 y residuo de 47 cc. No presenta incontinencia ni con esfuerzos. Continencia hasta 4-5 horas. A veces mínima pérdida que trata con salva-slip.

\section{DISCUSIÓN}

La lesión del tracto urinario inferior debe ser sospechada en mujeres traumatizadas con fractura pélvica, sangrado por introito vaginal, edema de vulva, retención urinaria, y en los casos en los que no se consiga colocar sonda vesical. Estas lesiones suelen ser causadas por grandes traumatismos por accidentes de tráfico, atropellos, o caídas de altura. Es más frecuente la lesión de la vulva y de la vagina representando la rotura uretral un acontecimiento poco frecuente y escasamente documentado, habiéndose reportado no más de 60 casos hasta la fecha ${ }^{1-9}$. En cualquier caso este tipo de lesiones deben de ser sospechadas y tratadas prontamente para evitar la aparición de fístulas uretrovaginales, y estenosis uretrales y vaginales.

La escasa incidencia de rotura traumática de uretra femenina se intenta explicar como debida a su escasa longitud y su importante movilidad, en contraposición a la porción membranosa de la uretra masculina, mucho más frecuentemente afectada por este tipo de traumatismos. Además su localización bajo el arco púbico le confiere cierta protección. Como mecanismo de fractura se definen varias teorias: la compresión de la uretra contra el labio postero-inferior del arco púbico durante el traumatismo y el retroceso de este último causaría avulsión de la uretra $^{1,2}$, la compresión lateral del anillo pélvico que resulta en un aumento de la distancia anteroposterior. La vejiga es elevada causando estiramiento y posterior

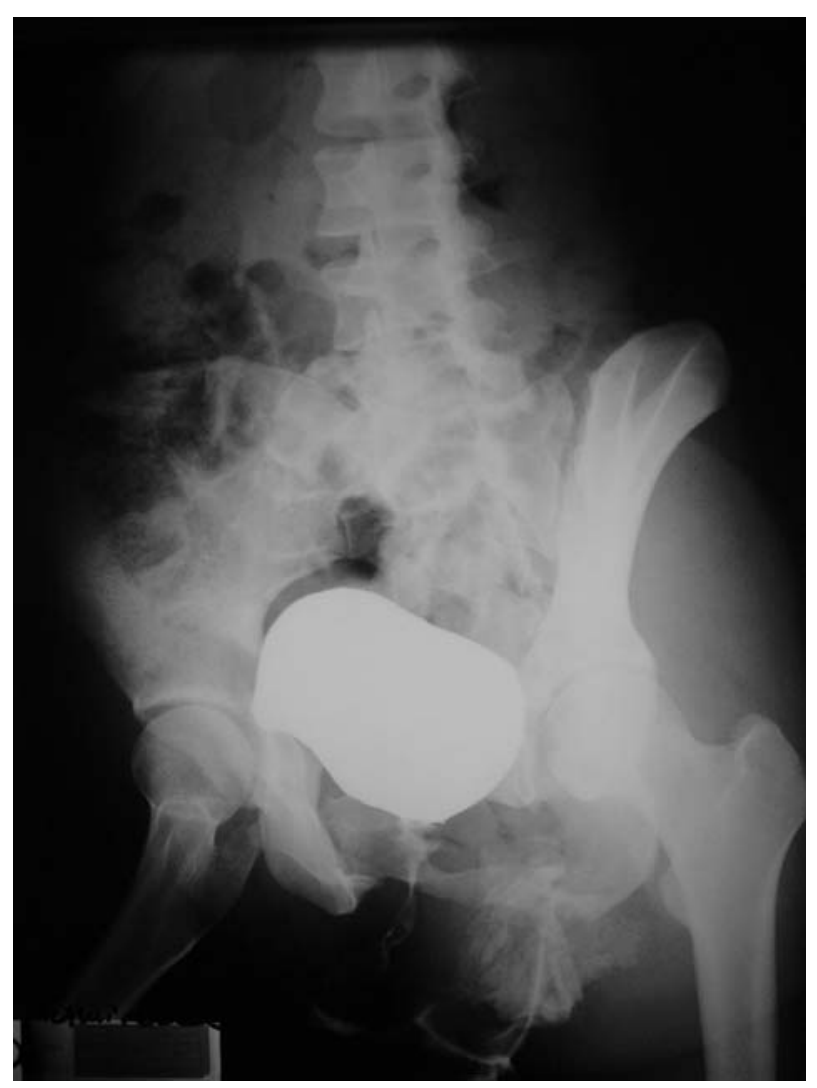

FIGURA 6. Cistografia post-intervención. No fugas de contraste. Cuello vesical integro. 
avulsión uretral. El tercer mecanismo sería la diastasis brusca del pubis resultando en una rotura de los ligamentos pubo-uretrales posteriores y del diafragma urogenital. En este caso la uretra se tornearía hacia posición anterior y posterior extendiéndose la rotura hacia el cuello vesical ${ }^{2}$. El primer mecanismo descrito podría asociarse a los dos últimos $^{2,10}$. Debido a la íntima relación de la uretra con la cara anterior de la vagina, las roturas de la primera casi siempre van asociadas a lesiones de la segunda, siendo frecuente la existencia de fístulas uretrovaginales. No es extraño que ante la llegada a urgencias de una mujer sangrando por vagina tras un accidente, con algún desgarro en la misma se avise al ginecólogo, quien sutura el desgarro sin atender al aparato urinario.

En cuanto a la variabilidad con la edad de este evento, aunque existen autores que no refieren preponderancia de una edad, una revisión exhaustiva de la literatura revela que niñas menores de 17 años sobrepasaban a adultos en una proporción de $33 / 7$. Una posible explicación sería la mayor compresibilidad de una pelvis todavía no osificada ${ }^{3}$.

En cuanto a los distintos tipos de rotura tenemos varias posibilidades, cada una de las cuales requería diferentes manejos: rotura circunferencial total de uretra distal, media o proximal, rotura circunferencial parcial anterior o posterior (casi siempre asociada a rotura vaginal), rotura longitudinal de tramo distal, medio, proximal, o total, etc. En las roturas de uretra proximal, casi siempre existe rotura asociada del cuello vesical, y lesión esfinteriana, con las consecuencias negativas que tendrá para la continencia posterior.

Decíamos al principio que es muy importante un diagnóstico precoz de estas lesiones que en ocasiones pueden pasar desapercibidas. El diagnóstico tardío puede tener graves consecuencias $^{1,3,5}$, desde una estenosis vaginal y hematocolpos posterior (en la pubertad), hasta fascitis necrotizantes y la posibilidad de desarrollar una enfermedad inflamatoria pélvica que desemboque en la desgraciada necesidad de una histerectomía. Por todo esto nos reiteramos, independientemente de que el manejo vaya a ser primario o diferido, en la necesidad de un buen y precoz diagnóstico, en el cual será necesario un estudio con contraste (urograma intravenoso o uretrocis- tografia bien por talla suprapúbica o retrógrada), además del resto de pruebas urgentes que requiera su estado clínico. Se comenta en diferentes trabajos ${ }^{1,3,5,6,8}$, la necesidad de una buena exploración ginecológica y perineal bajo anestesia que nos permita descartar no sólo lesiones uroginecológicas, sino también anales y rectales a menudo asociadas en estos grandes traumatismos. Pensamos que debe hacerse pero siempre que sea posible, sin olvidar que este tipo de pacientes suelen llegar al hospital graves e inestables por lo que no siempre es posible una exploración reglada al principio.

En lo referente al manejo de estas lesiones nos encontramos con la misma controversia que sucede en el varón. Existen tres modos distintos de enfoque:

1. Sondaje endoscópico inicial realineando la uretra rota para permitir posterior acercamiento de tejidos por sí mismos.

2. Colocación de talla suprapúbica y reparación diferida de la lesión uretral.

3. Reparación primaria.

Es importante tener en cuenta que un alto porcentaje de estas pacientes acude al hospital en situación grave, en ocasiones crítica, que nos impide realizar procedimientos laboriosos. Por tanto, la primera medida será la colocación de una talla suprapúbica si la paciente no orina, o si existe fístula vésico-vaginal, siendo reparada precozmente la lesión vaginal que suele ser menos dificultoso. Por otra parte, no hay que olvidar que la colocación de dicho drenaje suprapúbico es una arma no sólo terapéutica sino también diagnóstica, que nos permitirá la realización de una cistografia para saber la localización de la rotura y valorar el resto de la vejiga. En el primer caso que presentamos tenemos un ejemplo claro: la paciente es remitida de otro centro, lleva 3 meses con talla vesical y se le realiza una cistografia que nos define el problema, además de evitarle a la paciente una mayor morbilidad derivada de un hipotético acto urológico precoz cuando su estado era muy grave.

Para ilustrar un ejemplo de realineamiento endoscópico presentamos nuestro segundo caso. El sondaje inicial (en los primeros dias) con guía cistoscópica nos permite el teórico realineamiento de la uretra en aras de recuperar en lo posible la continuidad anatómica. En cualquier caso esta 
sería una técnica a utilizar en caso de mínimas roturas uretrales. En nuestro caso, que se trataba de una rotura uretral importante con rotura asociada del cuello vesical, este procedimiento no es efectivo. Algún autor refiere la aparición de estenosis uretrales secundarias ${ }^{6,10,12}$.

Por último, algunos autores recomiendan la cirugía primaria de estas lesiones ${ }^{1,3,6,11}$. Se basan en las siguientes premisas: la reparación primaria permite realineamiento temprano de la lesión uretral o vésico-uretral, y reparación de la lesión vaginal. Esto reduce la incidencia de estenosis uretrales y fístulas uretro-vaginales. La reparación vaginal primaria reduce asimismo la incidencia de estenosis vaginales. Algún autor refiere que la derivación urinaria primaria con cistostomía tiene un elevado índice de estenosis uretrales, además de cierre y pérdida del tejido uretral embebido en tejido cicatricial de muy difícil disección diferida. Además la uretra en la mujer tiene un papel primordial en la continencia, por lo que pérdidas de longitudes importantes de uretra pueden conducir a la incontinencia ${ }^{3}$. En cualquier caso, aunque estos autores afirman que la reconstrucción secundaria de la uretra ofrece unos grados razonables de continencia, ésta nunca es tan efectiva como la observada en el mecanismo natural de la uretra normal, es decir, tras reparación primaria. En todo caso en la revisión de la literatura que hemos realizado ${ }^{1,3-}$ 6,11 , existen varios conceptos que limitan este manejo primario: lesiones uretrales extensas o de toda la uretra, asociación de grandes fracturas pélvicas que alteran el campo anatómico, inestabilidad de la paciente y lesiones de órganos vitales, la presencia de un urólogo de guardia que no siempre es el de más experiencia en este campo, $\mathrm{y}$, como ocurrió en nuestro primer caso, el hecho de que muchas de estas pacientes llegan a los centros de referencia una vez están estables y curadas de lesiones más graves, por lo que han pasado semanas o meses desde el episodio. Todo esto hace que la elección más correcta sea en muchos casos una derivación suprapúbica y cirugía diferida por un equipo experimentado.

Nosotros recordamos los dos casos clínicos tratados en nuestro servicio, y documentados como tales en los últimos 28 años, lo que demuestra su escasa frecuencia. Observamos diferentes tipos de manejo en cada uno de ellos y en ambos una buena evolución. En el segundo caso, en el que existe una mínima incontinencia (apenas referida por la paciente) la lesión afectaba al cuello vesical que es lo que condiciona estas mínimas pérdidas.

Para concluir, varios conceptos: diagnóstico precoz en urgencias, manejo primero del estado de la paciente y procedimiento urológico lo menos laborioso si la enferma está en mal estado, importancia de las pruebas de imagen y buen pronóstico si el manejo es el correcto.

\section{REFERENCIAS}

1. OKUR H, KUÇUCAYDIN M, KAZEZ A, TURAN C, BOZKURT A.: Genitourinary tract injuries in girls. Br J Urol 1996; 78: 446-449.

2. PATIL U, NESBITT R, MEYER R.: Genitourinary tract injuries due to fracture of the pelvis in females: sequelae and their management. $\mathrm{Br} J$ Urol 1982; 54: 32-38.

3. HEMAL AK, DORAIRAJAN LN, GUPTA NP.: Posttraumatic complete and partial loss of urethra with pelvic fracture in girls: an apraisal of management. J Urol 2000; 163: 282-287.

4. AHMED S, NEEL KF.: Urethral injury in girls with fractured pelvis following blunt abdominal trauma. Br J Urol 1996; 78: 450-453.

5. PERRY MO, HUSMANN DA.: Urethral injuries in female subjects following pelvic fractures. $J$ Urol 1992; 147: 139-143.

6. THAMBI DORAI CR, BOUCAUT HAP, DEWAN PA.: Urethral injuries in girls with pelvic trauma. Eur Urol 1993; 24: 371-374.

7. CARTER CT, SCHAFER N.: Incidence of urethral disruption in females with traumatic pelvic fractures. Am J Emerg Med 1993; 11: 218-220.

8. NETTO JUNIOR NR, IKARI O, ZUPPO VP.: Traumatic rupture of female urethra. Urology 1983; 22: 601-603.

9. MEVEL O, TAHAN H, MICHEL F.: Rupture of the female uretra in pelvic fractures: report of two cases. Prog Urol 2000; 10: 1212-1216.

10. CASSELMAN RC, SCHILLINGER JF.: Fractured pelvis with avulsion of the female urethra. $J$ Urol 1976; 117: 385-386.

11. PODESTÁ ML, JORDAN GH.: Pelvic fracture urethral injuries in girls. J Urol 2001; 165: 1660-1665.

12. WATWERHOUSE K, GROSS M.: Trauma to the genitourinary tract: a five years experience with 251 cases. J Urol 1969; 101: 241-246.

Dr. A. Blanco Díez

Avda. Montserrat, $18-6^{\circ} \mathrm{E}$

15009 La Coruña

(Trabajo recibido el 1 julio de 2003) 